\title{
Long-term eculizumab improves clinical outcomes in atypical hemolytic uremic syndrome
}

\author{
Ramon Vilalta • Enrique Lara • Alvaro Madrid • \\ Sara Chocron • Marina Muñoz • Alex Casquero • \\ Jose Nieto
}

Received: 17 February 2012 /Revised: 3 July 2012 / Accepted: 4 July 2012 / Published online: 14 August 2012

(C) The Author(s) 2012. This article is published with open access at Springerlink.com

\begin{abstract}
Background Atypical hemolytic uremic syndrome (aHUS) is a rare genetic disorder caused by chronic uncontrolled complement activation.

Case-diagnosis/treatment We present a 4-year-old girl with aHUS who had multiple severe clinical manifestations of thrombotic microangiopathy (TMA) including acute kidney injury, dilated cardiomyopathy, and cardiorespiratory arrest. She was managed with intensive plasma exchange and hemodialysis, which could not halt the progression of TMA. The initial single dose of eculizumab only temporarily improved the clinical symptoms of TMA. Sustained improvement of renal, hematological, and cardiac values were only achieved upon institution of chronic treatment with eculizumab. During long-term treatment with eculizumab ( $>2.5$ years), she has had no further clinical manifestations of TMA, and required neither plasma exchange nor hemodialysis.

Conclusion Chronic eculizumab treatment was associated with control of complement-mediated TMA and sustained long-term improvement in renal and cardiac function.
\end{abstract}

\section{Background}

Atypical hemolytic uremic syndrome (aHUS) is a rare genetic disorder caused by chronic uncontrolled complement activation $[1,2]$. Although complement mutations have been found in $50-70 \%$ of patients with aHUS, identification of a genetic mutation is not necessary for diagnosis or treatment initiation

R. Vilalta $(\bowtie) \cdot$ E. Lara $\cdot$ A. Madrid $\cdot$ S. Chocron $•$ M. Muñoz

A. Casquero $\cdot$ J. Nieto

Pediatric Nephrology Department,

Hospital Universitari Vall d'Hebron,

Pg. Vall d'Hebron 129-139,

08039 Barcelona, Spain

e-mail: rvilaltacasas@gmail.com
[3]. aHUS is characterized by systemic thrombotic microangiopathy (TMA) and multiple organ damage, which result in significant morbidity and mortality [4]. Despite supportive management, including plasma exchange/infusion (PE/PI), $33-40 \%$ of patients progress to end-stage renal disease (ESRD) or die at the first clinical manifestation $[1,4,5]$. Thus, there remains a need for new therapeutic strategies [6].

Eculizumab is a humanized monoclonal antibody to terminal complement protein $\mathrm{C} 5$ that prevents activation of the terminal complement pathway by binding $\mathrm{C} 5$ and inhibiting generation of pro-inflammatory C5a and the lytic C5b-9 membrane-attack complex. It is licensed for treatment of paroxysmal nocturnal hemoglobinuria (PNH) and aHUS (Soliris ${ }^{\circledR}$ SmPC, Alexion Europe). We report on a severely ill child with aHUS treated with eculizumab. The patient was initially managed with intensive PE, but clinical manifestations of aHUS were observed, including laboratory evidence of TMA and organ damage. A single dose of eculizumab initially led to marked improvement in renal function, but the clinical manifestations of TMA were again seen after 6 weeks. Chronic eculizumab treatment was introduced to maintain complete blockade of complement activation, which correlated with clinical improvement (Soliris ${ }^{\circledR}$ SmPC).

\section{Case report}

A severely ill 1-year-old Caucasian girl with a several-day history of vomiting and refusal to eat presented on 31 December 2008 with hemolytic anemia, thrombocytopenia, acute renal insufficiency, and intussusception. Schistocytes on peripheral blood film, elevated lactate dehydrogenase (LDH) $2674 \mathrm{U} / 1$ (normal range: 216-360 U/1), decreased platelets $108 \times 10^{9} / 1$ (normal range: $150-350 \times 10^{9} / 1$ ), 
elevated creatinine $221 \mu \mathrm{mol} / 1$ (normal range: $35-55 \mu \mathrm{mol} /$ 1) and urea $234 \mathrm{mg} / \mathrm{dl}$ (normal range: $7-21 \mathrm{mg} / \mathrm{dl}$ ), and reduced hemoglobin $(\mathrm{Hb}) 6.5 \mathrm{mg} / \mathrm{dl}$ (normal range: 10 $14 \mathrm{mg} / \mathrm{dl}$ ) and albumin $2.76 \mathrm{~g} / \mathrm{dl}$ (normal range: $3.5-5.5 \mathrm{~g} /$ dl) were detected. Activity of ADAMTS-13 (Von Willebrand factor-cleaving protease) was within the normal range $(83 \%)$, thus excluding thrombotic thrombocytopenic purpura (TTP) [7]. Complement levels were within the normal range: $\mathrm{C} 3,94 \mathrm{mg} / \mathrm{dl}$ (normal range: $85-120 \mathrm{mg} / \mathrm{dl}$ ); $\mathrm{C} 4$, $16 \mathrm{mg} / \mathrm{dl}$ (normal range: $15-40 \mathrm{mg} / \mathrm{dl}$ ). As stool cultures for Shigella, Salmonella, Yersinia, and Candida were all negative, she was diagnosed with aHUS. Initial management comprised multiple blood transfusions, intensive plasma exchange (2-3 sessions per week), and hemodialysis. These interventions initially improved diuresis, the patient's creatinine decreasing to a minimum of $94 \mu \mathrm{mol} / \mathrm{l}$, but she continued to be hospitalized, to need repeated blood transfusions, have severe proteinuria $(233 \mathrm{mg} / \mathrm{dl})$, and suffer from hypertension (blood pressure increasing to 128/ $95 \mathrm{mmHg}$ in the days following presentation) and edema. During the following weeks, she also suffered multiple catheter infections and had to maintain a nasogastric tube for feeding. Overall, the clinical status of the patient further deteriorated, presenting 17 February 2009 with acute renal insufficiency, severe anemia, macroscopic hematuria, proteinuria, arterial hypertension, and dilated cardiomyopathy. Laboratory values showed decreased platelet count $(132 \times$ $\left.10^{9} / 1\right)$, an elevated LDH of $1740 \mathrm{U} / 1$ and continued anemia $(\mathrm{Hb} 7.1 \mathrm{mg} / \mathrm{dl})$, and elevated serum creatinine $(187 \mu \mathrm{mol} / \mathrm{l})$. The child also experienced cardiorespiratory arrest requiring cardiopulmonary resuscitation and mechanical ventilation. Genetic analysis revealed a mutation in the $\mathrm{C}$-terminal region of complement factor $\mathrm{H}$ (CFH) (a novel heterozygous mutation; $3355 \mathrm{G}>\mathrm{A}$; Asp1119Asn; SCR19), with normal membrane cofactor protein (MCP) and Factor I, and no CFH autoantibodies. PE (fresh-frozen plasma, 2-3 times per week) was continued for the next 3 months, with blood transfusions given as required. The patient's anti-hypertensive therapy (hydralazine, nifedipine, labetalol, and urapidil) was also continued (Fig. 1). She experienced pulmonary edema in March and again in May. Myocardial dysfunction was diagnosed and an ejection fraction of $32 \%$ (normal values: $56-78 \%$ ) was measured in May. Due to concerns that this was caused by fluid overload from PE, PE and hemodialysis were tapered. This led to an improvement in renal function, but she continued to receive antihypertensive treatment and multiple blood transfusions (Fig. 1).

In July, when the patient presented again with cardiac dysfunction and an ejection fraction of $31 \%$, with moderately controlled blood pressure (100/85) and not managed with $\mathrm{PE}$, the decision was made to initiate treatment with eculizumab; she received one 600-mg eculizumab infusion (at this time, the patient was vaccinated against Neisseria meningococcus serotypes $\mathrm{A}$ and $\mathrm{Cm}$, and antibiotic prophylaxis with penicillin was provided). Eculizumab treatment led to normalization of renal and ventricular function, stabilization of hematologic parameters, and blockade of terminal complement activity (Fig. 1).

As the eculizumab dose was not repeated, and terminal complement blockade not maintained, TMA complications were seen again after 8 weeks (mid-September 2009), when the patient presented with renal insufficiency (creatinine, $141 \mu \mathrm{mol} / \mathrm{l})$ and evidence of hemolysis and platelet consumption (LDH, $760 \mathrm{U} / 1$; platelet count, $\left.151 \times 10^{9} / 1\right)$. Chronic eculizumab treatment was initiated following the recommended dosing by weight $(5-10 \mathrm{~kg})$ (Soliris ${ }^{\circledR}$ SmPC): initial dose $600 \mathrm{mg}$, followed by $300 \mathrm{mg}$ every 2-3 weeks. At this time, the patient's antihypertensive treatment was stopped. Creatinine started to improve prior to eculizumab initiation but levels normalized after 6 days of resuming eculizumab treatment (Fig. 1) and cardiac function improved. LDH levels stabilized but remained elevated (Fig. 1); the complement activity assay showed immediate, sustained blockade of terminal complement activation; and platelet counts remained within the normal range (Fig. 1). The elevated LDH was not the result of anemia, as Hb levels were normal $(10 \mathrm{mg} / \mathrm{dl})$ and no schistocytes were seen, with no further need for RBC transfusions.

At last follow-up visit (14 November 2011; $>2.5$ years since admission), the patient remained on chronic eculizumab treatment; no new clinical complications of TMA have occurred while receiving treatment, and no blood transfusion, plasma exchange, or hemodialysis has been necessary. Serum creatinine is $26.5 \mu \mathrm{mol} / \mathrm{l}$, and serum protein $34 \mathrm{mg} /$ dl, equivalent to $12 \mathrm{mg} / \mathrm{m}^{2} / \mathrm{h}$. No further indication of hypertension or cardiac insufficiency has been noted and her ejection fraction has been normalized (64-70\%). The patient has gained $6 \mathrm{~kg}$ of weight and her overall health has improved and normalized entirely. She shows a completely normal development and full recovery of a good quality of life as reported by her parents.

During the $>2.5$-year period of chronic eculizumab treatment, measurements of complement activity have shown complete terminal complement blockade, which corroborate the clinical data of no manifestations of TMA (Fig. 1).

\section{Discussion}

PE has been used historically for the management of aHUS [1], but in the present case, progressive renal deterioration and an increased need for blood transfusions was seen despite PE (2-3 sessions per week for 16 weeks). Progressive cardiac dysfunction was also detected. Blockade of the terminal complement protein $\mathrm{C} 5$ with eculizumab is a rational treatment for aHUS that directly targets chronic 
Fig. 1 Response to eculizumab in a 1-year-old female with atypical hemolytic uremic syndrome (aHUS), as shown by creatinine levels, platelet counts, LDH, hemolysis assay of complement activation [unpublished data], and body weight and height. Initial plasma exchange and hemodialysis was started at the time of diagnosis, but the child experienced clinical manifestations of thrombotic microangiopathy (TMA) after approximately 7 weeks. Following cessation of plasma exchange, a single dose of eculizumab $600 \mathrm{mg}$ was given (green arrow), which resulted in stabilization of renal and hematologic function. After approximately 8 weeks, clinical manifestations became apparent again as no complement inhibition was present, after which continuous eculizumab treatment was reinitiated (initial dose $600 \mathrm{mg}$, followed by $300 \mathrm{mg}$ every 2-3 weeks). Dashed lines show the normal range for creatinine, platelets and $\mathrm{LDH}$, and threshold for complete complement blockade

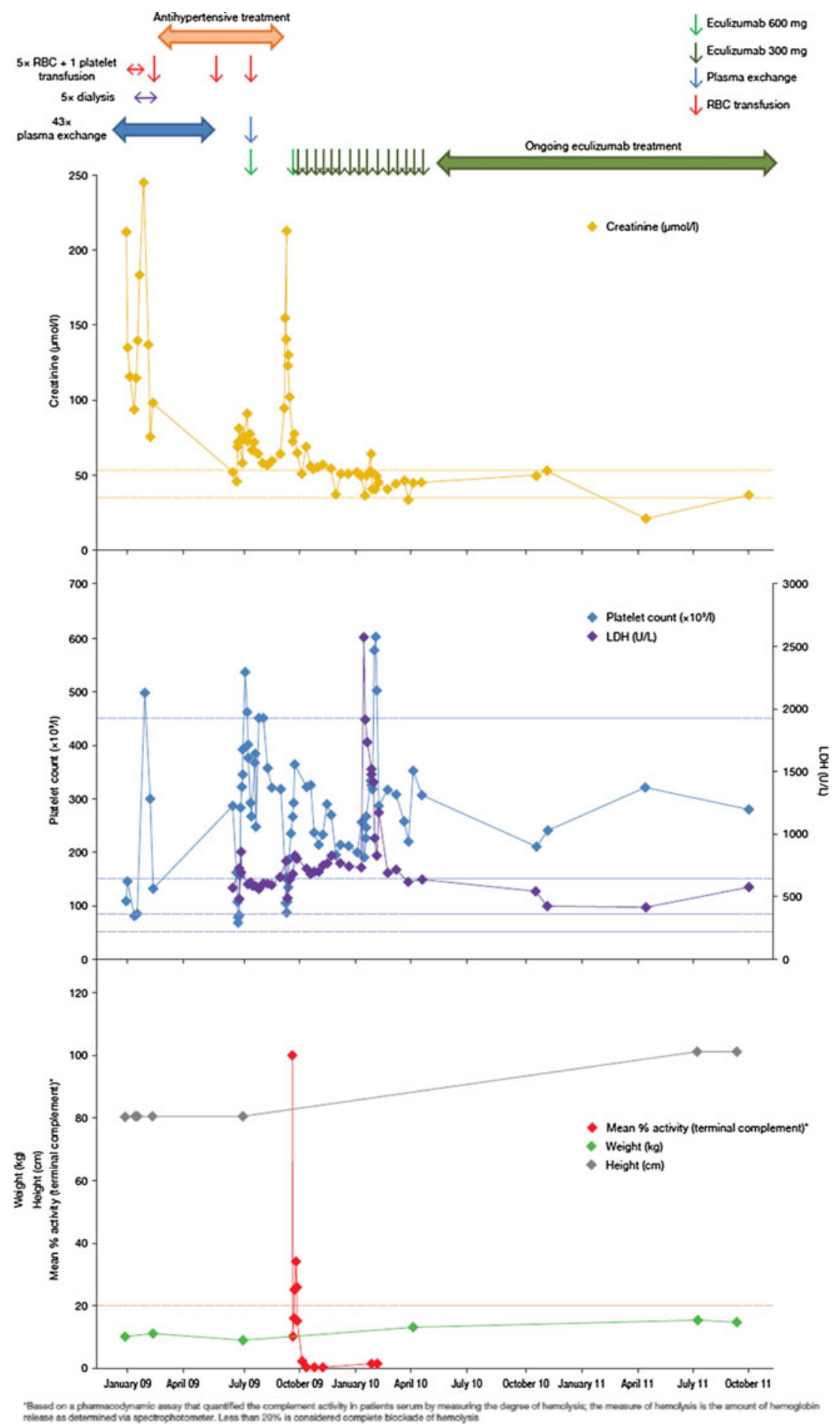

uncontrolled complement activity [6]. As demonstrated by the present case, a single infusion can have an immediate effect, but can only achieve terminal complement blockade for a short duration. A reduced dose or discontinuation of eculizumab treatment has previously been shown to lead to rapid deterioration in organ function $[6,8,9]$. Indeed, in the recent aHUS clinical trials with eculizumab, five of 18 patients who discontinued eculizumab experienced severe
TMA complications following the missed eculizumab dose (Soliris ${ }^{\circledR} \mathrm{SmPC}$ ).

In this patient, following discontinuation of eculizumab, reinitiation of eculizumab therapy rapidly restored the clinical and biological parameters. However, several cases have been reported in literature where this has not been possible and reinitiation of eculizumab therapy following discontinuation cannot be counted on to salvage organ function [6] 
This patient maintained an elevated LDH (a measure of tissue damage and/or hemolysis), although the level stabilized during eculizumab treatment. The elevated level may be the result of organ damage, as no further anemia and thrombocytopenia were detected. Complement activation can be further amplified by common triggers such as infection. In our patient, this manifested as a large, transient increase in LDH during a respiratory tract infection. However, despite this increase, there was no evidence of complement-mediated TMA during eculizumab treatment; renal function remained stable and hematologic function was rapidly normalized without a requirement for blood transfusions.

In contrast to PE, eculizumab treatment inhibits the underlying process of chronic uncontrolled complement activation. Thus, chronic eculizumab treatment permanently suppresses clinical manifestations of TMA. Cardiac function markedly improved in this patient during eculizumab treatment. The acute cardiac failure in February 2009 could have been caused by fluid overload due to PE and high BP. However, the persistent cardiomyopathy could not have been caused by elevated blood pressure or fluid overload alone as cardiomyopathy persisted well after PE had been terminated (April 2009) and blood pressure was fairly controlled, and did not improve until after chronic eculizumab therapy was initiated, thus suggesting TMA could be one of the causes. No histological studies of the myocardium were, however, conducted.

Long-term inhibition of chronic uncontrolled complement activation and complement-mediated TMA was accompanied by general improvement in the child's clinical condition, including appropriate weight gain and a lack of subsequent events. The child has been treated for more than 2.5 years and treatment is well tolerated.

In conclusion, these findings are consistent with previous case reports of eculizumab in patients with aHUS, in which treatment was associated with control of complementmediated TMA $[6,8,9]$. In addition, terminal complement blockade with eculizumab was directly correlated with sustained, long-term ( $>2.5$ years) marked improvements in renal and cardiac function. Interruption of eculizumab treatment led to a rapid return of the clinical manifestations of TMA, but improvement and stabilization of organ function and hematological values was restored upon reinitiation of therapy in this case.
Acknowledgments Medical writing support was provided by Åsa Lommelé (Alexion Pharma International) and Bioscript Stirling Ltd (London, UK), funded by Alexion Pharmaceuticals.

Transparency declarations Dr. Ramon Vilalta has sat on advisory boards for Alexion. The other authors have no potential conflicts of interest to declare.

Open Access This article is distributed under the terms of the Creative Commons Attribution License which permits any use, distribution, and reproduction in any medium, provided the original author(s) and the source are credited.

\section{References}

1. Caprioli J, Noris M, Brioschi S, Pianetti G, Castelletti F, Bettinaglio P, Mele C, Bresin E, Cassis L, Gamba S, Porrati F, Bucchioni S, Monteferrante G, Fang CJ, Liszewski MK, Kavanagh D, Atkinson JP, Remuzzi G, International Registry of Recurrent and Familial HUS/TTP (2006) Genetics of HUS: the impact of MCP, CFH, and IF mutations on clinical presentation, response to treatment, and outcome. Blood 108:1267-1279

2. Besbas N, Karpman D, Landau D, Loirat C, Proesmans W, Remuzzi G, Rizzoni G, Taylor CM, Van de Kar N, Zimmerhackl LB, European Paediatric Research Group for HUS (2006) A classification of hemolytic uremic syndrome and thrombotic thrombocytopenic purpura and related disorders. Kidney Int 70:423-431

3. Loirat C, Frémeaux-Bacchi V (2011) Atypical hemolytic uremic syndrome. Orphanet J Rare Dis 6:60

4. Noris M, Caprioli J, Bresin E, Mossali C, Pianetti G, Gamba S, Daina E, Fenili C, Castelletti F, Sorosina A, Piras R, Donadelli R, Maranta R, van der Meer I, Conway EM, Zipfel PF, Goodship TH, Remuzzi G (2010) Relative role of genetic complement abnormalities in sporadic and familial aHUS and their impact on clinical phenotype. Clin J Am Soc Nephrol 5:1844-1859

5. Taylor CM, Chua C, Howie AJ, Risdon RA, British Association for Paediatric Nephrology (2004) Clinico-pathological findings in diarrhoea-negative haemolytic uraemic syndrome. Pediatr Nephrol 19:419-425

6. Zuber J, Le Quintrec M, Sberro-Soussan R, Loirat C, FrémeauxBacchi V, Legendre C (2011) New insights into postrenal transplant hemolytic uremic syndrome. Nat Rev Nephrol 7:23-35

7. Bianchi V, Robles R, Alberio L, Furlan M, Lammle B (2002) Von Willebrand factor-cleaving protease (ADAMTS13) in thrombocytopenic disorders: a severely deficient activity is specific for thrombotic thrombocytopenic purpura. Blood 100:710-713

8. Châtelet V, Lobbedez T, Frémeaux-Bacchi V, Ficheux M, Ryckelynck JP, Hurault de Ligny B (2010) Eculizumab: safety and efficacy after 17 months of treatment in a renal transplant patient with recurrent atypical hemolytic-uremic syndrome: case report. Transpl Proc 42:4353-4355

9. Rinder CS, Rinder HM, Smith BR, Fitch JC, Smith MJ, Tracey JB, Mathis LA, Squinto SP, Rollins SA (1995) Blockade of C5a and C5b-9 generation inhibits leukocyte and platelet activation during extracorporeal circulation. J Clin Invest 96:1564-1572 